\title{
Statistical Regression based Rotation Estimation Technique of Color Image
}

\author{
Joydev Hazra \\ Department of Information Technology \\ Heritage Institute of Technology \\ West Bengal, India
}

\author{
Aditi Roy Chowdhury \\ Department of Computer Science and Technology \\ BPCIT, Krishnagar \\ West Bengal,India
}

\author{
Paramartha Dutta \\ Department of Computer and System Science \\ Visva-Bharati University, Santiniketan \\ West Bengal, India
}

\begin{abstract}
- This paper describes a rotational angle estimation of different color images. This estimation method is primarily based on weighted linear regression lines of the three color components of a color image as well as the influence of each component. Preservation of the chromatic information makes this method helpful to efficiently calculate the rotational angle between the referenced and sensed image pair. The experiments justify that the proposed method is robust ensuring its applicability to any kind of color images.
\end{abstract}

\section{General Terms:}

Image Transformation, Color image components

\section{Keywords:}

Weighted Linear Regression Line, Composite Rotation

\section{INTRODUCTION}

Image alignment is a technique to align two images, reference (fixed) image and the sense (changed) image primarily used in image registration. Sense image is basically the image of the same scene as that of reference but taken at different times or from different viewpoints. It has great importance in medical diagnosis and treatment, in image sensing and especially in computer vision. Rotation estimation is one of the important necessity in image alignment.

Over the years, research on image registration and image transformation has produced a lot of different methods. Many methods use different feature matching techniques like Area based and Feature based [1], [2]. In Area based methods, frames of predefined size or even entire images are used for the corresponding estimation [3], [4], [5]. Area based methods may be mutual information [6] oriented, correlation coefficient [7] oriented, Fourier transform [8], [9] oriented etc. On the contrary, Feature based methods consider control points [10], [6], graph matching algorithm [11], clustering technique [12]. But algorithms that are directly applicable on color images are very rare in literature. In many cases, color images are directly converted to gray scale image and then the registration algorithm are applied on that gray scale version. But due to this conversion, many chromatic information about the color image are lost. In QPOC [13], the authors proposed a subpixel registration algorithm for color images. This is an extension of the classical POC [14]. QPOC correlation coefficients can estimate the spatial shifts among the images. Registration technique based on physical forces [15] also directly applies on color images. It is basically a Feature based method as it implies the similarity criterion and the optimization method simultaneously. In quaternion phase correlation [16], full chromatic information is used for image registration. In this article, we use a weighted linear regression method to identify the rotational angle between the reference and the sense color images. Statistically line of regression offers the index of symmetry of an image content. Motivated by the prospect of preserving chromatic information in color images, this paper is an extension of previous work [17]. Proposed method performs rotational angle estimation of color images at global level. It is fully unsupervised and is applicable to any type of image.

The paper is organized as follows. The evaluation procedure is described in detail in Section 2. Section 3 presents results and discussion. Section 4 contains conclusion.

\section{PROPOSED METHOD}

The proposed algorithm consists of two stages: centroid calculation and Line of regression detection.

\subsection{Foundation}

An image of size $M \times \mathrm{N}$ can be expressed as two-dimensional function of the form $f(x, y)$, where $1 \leq x \leq M, 1 \leq y \leq N$. The weighted centroid $\left(C_{x}, C_{y}\right)$ of each color component of the image can be computed [17]. We calculate the line passing through the centroid and yielding minimum weighted sum of all the image pixels falling perpendicularly upon the line for each of the above mentioned color components. This line provides the line of regression of each component of the color object passing through their 
respective centroids. Here weights being the corresponding pixel intensities. Thus, we find

$$
\begin{gathered}
\min _{m, c} \sum_{x=1}^{M} \sum_{y=1}^{N} f(x, y) \frac{(y-\overline{m x+c})^{2}}{\left(1+m^{2}\right)} \\
\text { Let, } \phi=\sum_{x=1}^{M} \sum_{y=1}^{N} f(x, y) \frac{(y-\overline{m x+c})^{2}}{\left(1+m^{2}\right)}
\end{gathered}
$$

Equation 22 is minimum if $\frac{\partial \phi}{\partial m}=0$ and $\frac{\partial \phi}{\partial c}=0$ and the Hessian

$$
\left(\begin{array}{cc}
\frac{\partial^{2} \phi}{\partial m^{2}} & \frac{\partial^{2} \phi}{\partial m \partial c} \\
\frac{\partial^{2} \phi}{\partial c \partial m} & \frac{\partial^{2} \phi}{\partial c^{2}}
\end{array}\right)
$$

is positive definite at $(\hat{m}, \hat{c})$. Solving $\frac{\partial \phi}{\partial m}=0$ and $\frac{\partial \phi}{\partial c}=0$ at $(\hat{m}, \hat{c})$ we get,

$$
\left(\begin{array}{c}
\hat{m} \\
\hat{c}
\end{array}\right)=A\left(\begin{array}{c}
\sum_{x=1}^{M} \sum_{y=1}^{N} f(x, y) x y \\
\sum_{x=1}^{M} \sum_{y=1}^{N} f(x, y) y
\end{array}\right)
$$

where,

$$
A=\left(\begin{array}{c}
\sum_{x=1}^{M} \sum_{y=1}^{N} f(x, y) x^{2} \sum_{x=1}^{M} \sum_{y=1}^{N} f(x, y) x \\
\sum_{x=1}^{M} \sum_{y=1}^{N} f(x, y) x \sum_{x=1}^{M} \sum_{y=1}^{N} f(x, y)
\end{array}\right)^{-1}
$$

Basically, the axis of symmetry divides the image into two regions $F_{1}$ and $F_{2}$ such that difference between the weighted sum of the two regions is minimum. i.e.,

$\Delta\left(F_{1}, F_{2}\right)=\left|\sum_{(x, y) \in F_{1}} f(x, y)-\sum_{(x, y) \in F_{2}} f(x, y)\right|$ is minimum.

where, $f(x, y)$ is the intensity of the image pixel at $(x, y)$. This minimum value of $\Delta($,$) is called index of symmetry of the corre-$ sponding image.

For any perfect symmetrical object this difference ideally becomes zero. Line of regression passing through centroid can be treated as the axis of symmetry of the image.

\subsection{Our Algorithm}

To solve the problem of rotational angle detection calculate the axes of symmetry of the reference and sense color images. Now, decompose pixel intensities of the original color image into red, green and blue components. The figure 1 shows the original image and its three color components. Then average intensities of red color component can be computed using equation 4

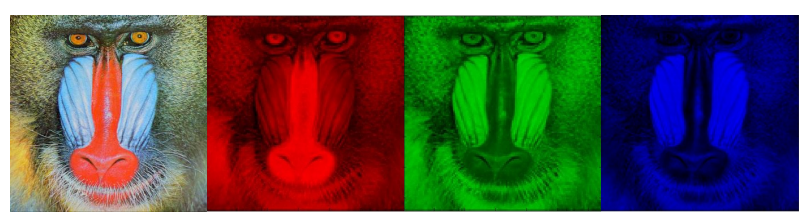

(a)

(b)

(c)

(d)

Fig. 1: (a) Baboon image. (b) Red component of (a). (c) Green component of (a). (d) Blue component of (a).

$$
\begin{aligned}
& \mu_{R}=A v(R) \\
& \mu_{G}=A v(G) \\
& \mu_{B}=A v(B)
\end{aligned}
$$

where $\theta \leq \mu_{R} \leq 255$ and $A v(R)$ function calculates the average intensity of red color component for all pixels of the image. This can be computed by summing the intensity values of each pixel in the image corresponding to any specific component and diving with the total number of pixels in the image. Similarly, we find the average values for green and blue components using equation 5 and 6 Let, $\pi_{R}, \pi_{G}, \pi_{B}$ indicate influence of each components of red, green and blue of any color image. Equation 78 and 9 represents $\pi_{R}, \pi_{G}, \pi_{B}$ as

$$
\begin{aligned}
\pi_{R} & =\frac{\mu_{R}}{\mu_{R}+\mu_{G}+\mu_{B}} \\
\pi_{G} & =\frac{\mu_{G}}{\mu_{R}+\mu_{G}+\mu_{B}} \\
\pi_{B} & =\frac{\mu_{B}}{\mu_{R}+\mu_{G}+\mu_{B}}
\end{aligned}
$$

The axis of symmetry of each color component of the referenced image makes an angle $\theta_{R}, \theta_{G}$ and $\theta_{B}$ with the horizontal line passing through centroid $\left(C_{x}, C_{y}\right)$. So, the axis of symmetry of the referenced image makes an angle $\theta$ with the horizontal line passing through centroid which can be represented as

$$
\theta=\pi_{R} \theta_{R}+\pi_{G} \theta_{G}+\pi_{B} \theta_{B}
$$

Similarly, he axis of symmetry of the sensed image can be calculated. So, the angular difference between the axes of symmetry of the referenced color image with that of the sensed color image, provides the angle of rotation of the sensed image with respect to the reference image. This algorithm can efficiently calculate the rotational angle in case of composite rotation. Experimental results shows that the computed rotational angle is very close to the original rotated angle.

\subsection{Computational Complexity}

Computational complexity of our algorithm for each component of any color image of size $N \times N$ is $\mathrm{O}\left(N^{2}\right)$. So, the total complexity to identify the rotational angle between the sensed and referenced color image is $\mathrm{O}\left(N^{2}\right)$.

\section{EXPERIMENT}

We apply our method on different types of color images. The method was tested on about 75 color images under different contexts.Here anticlockwise rotation is consider as positive rotation. Figure 2(a) is the original baboon image of size $350 \times 350$ and figure 2 b) shows its rotational counterpart by an angle 
Table 1. : Performance of our method for different images with different angle of rotation

\begin{tabular}{|c|c|c|c|c|}
\hline Original Figure & Rotation in degree & Result using our algorithm & $\begin{array}{c}\text { Influence of red, green } \\
\text { and blue components }\end{array}$ & Accuracy \\
\hline Baboon image(Fig. 2(a)) & $100^{0}$ (Fig. 2(b)) & $(242.9551-142.6155)=100.3396^{0}$ & $0.3623,0.3396,0.2981$ & $99.66 \%$ \\
\hline Peppers image(Fig. 2(c)) & $53.233^{0}$ (Fig. 2(d)) & $(132.3304-78.9765)=53.3539^{0}$ & $0.4511,0.3486,0.2004$ & $99.77 \%$ \\
\hline Lenna image(Fig. 3(a)) & $-39^{0}$ (Fig. 3(b)) & $(173.6623-212.6623)=-39^{0}$ & $0.4767,0.2849,0.2384$ & $100 \%$ \\
\hline Cat image(Fig. 3(c)) & $-123^{0}$ (Fig 3(d)) & $(78.8327-197.4949)=-122.6622^{0}$ & $0.3865,0.3378,0.2757$ & $99.72 \%$ \\
\hline
\end{tabular}

$\overline{\text { Algorithm } 1 \text { Rotational angle detection between sense color image }}$ $\mathrm{S}$ and reference color image $\mathrm{R}$

Ensure: Image $\mathrm{S}$ preserves the information of original image $\mathrm{R}$

1: Calculate weighted centroid of both images $\mathrm{S}$ and $\mathrm{R}$

2: Compute the weighted statistical regression line $Y=m_{S} X+$ $c_{S}$ for each component of the color image $S$ passing through the centriod $\left(C_{X}, C_{Y}\right)$.

3: This weighted statistical regression line makes for each color component an angle $\theta_{R}, \theta_{G}, \theta_{B}$ with the horizontal line passing through centroid

4: Calculate average intensities of each color components by equation 4,5 and 6

5: Calculate influence of each color components using equation 7. 8 and 9

6: The angle between regression line and the horizontal line passing through the centroid can be calculated using equation 10

7: Repeat steps 2 to 6 on color image $\mathrm{R}$

8: Find the angle $\theta$ between the two regression lines $\theta=\theta_{S}-\theta_{R}$

9: Angle $\theta$ is reported as the angle of rotation of color image

$100^{0}$. In original image $\pi_{R}=0.3623, \pi_{G}=0.3396, \pi_{B}=0.2981$ and $\theta_{R}=148, \theta_{G}=126$ and $\theta_{B}=155$. So $\theta=142.6155$. After rotation $\pi_{R}=0.3623, \pi_{G}=0.3396, \pi_{B}=0.2981$ and $\theta_{R}=248, \theta_{G}=227$, $\theta_{B}=255$. So $\theta=242.9551$. So, line of regression of original image makes an angle $142.6155^{\circ}$ with the horizontal line passing through the centroid. In figure 2 b) this line of regression makes an angle $242.9551^{0}$ with the horizontal line passing through the centroid. Thus the angle between these two lines is $100.3396^{\circ}$. Hence degree of disparity and relative disparity is $0.3396^{\circ}$ and $0.3396 \%$ respectively. So, it is observed that our method gives convincing result in this case. Again figures 2 (c) is the original peppers image of size $128 \times 128$ and 2 (d) shows rotation of the original image of peppers by an angle $53.233^{\circ}$ first (figure 2(c)). Using our method we find the angle as $53.3539^{\circ}\left(132.3304^{\circ}\right.$ for rotated image $-78.9765^{\circ}$ for original image). Hence disparity is $0.1209^{\circ}$ and $0.2271 \%$ respectively. Figure 3 a) shows Lena image of size $128 \times 128$. We rotate this image $-39^{\circ}$ (i.e. clockwise direction) which is represented in Figure 3 b). Using our method we can find that the difference between two lines of regression is $-39^{\circ}$. Again our proposed method offers actual value. Figure 3 (c) shows cat image of size $128 \times 128$. We rotate this image $-123^{\circ}$ (clockwise) which is represented in Figure 3 d). Using our method we can find that the difference between two lines of regression is $-122.6622^{\circ}$. Again our proposed method gives good result. Experimental results are recorded in Table 1

In case of composite rotation, we can also efficiently identify

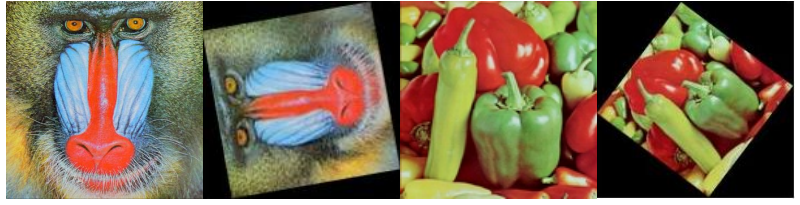

(a)

(b)

(c)

(d)

Fig. 2: (a) Baboon image. (b) After $100^{\circ}$ anticlockwise rotation of (a). (c) Peppers image. (d) After $53.233^{0}$ anticlockwise rotation of (c).

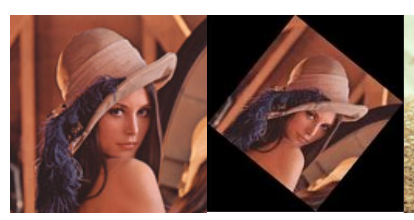

(a) (b)

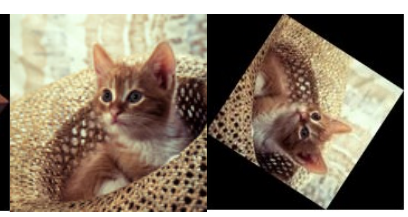

(c) (d)
Fig. 3: (a) Lena image. (b) After $39^{0}$ clockwise rotation of (a). (c) Cat image. (d) After $123^{0}$ clockwise rotation of (c).

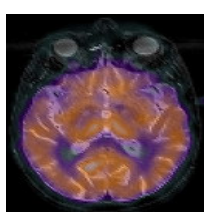

(a)

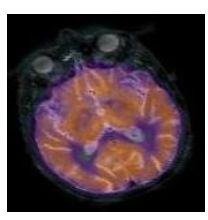

(b)

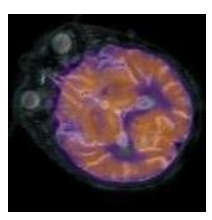

(c)
Fig. 4: (a) MRI image. (b) After $20^{\circ}$ anticlockwise rotation of (a). (c) After $40^{\circ}$ anticlockwise rotation of (b).

the rotation angle using our algorithm. In Figure 4 we rotate the MRI image at an angle $20^{\circ}$ and then again $40^{\circ}$. From our algorithm $\pi_{R}=0.3155, \pi_{G}=.2801$ and $\pi_{B}=.4044$ and $\theta_{R}=177, \theta_{B}=178$ and $\theta_{G}=178$. The line of regression of the referenced color image makes an angle $\theta_{o}=177.5956$ with the horizontal axis passing through centroid. For sensed image $\theta_{R}^{\prime}=237, \theta_{G}^{\prime}=238$ and $\theta_{B}^{\prime}=238$. So, for sensed image, this angle is $\theta_{r}^{\prime}=237.5956$. So, the angle between the two line of regressions is $60^{\circ}$. 


\section{SUMMARY}

This paper presents a technique to estimate two dimensional rotation in registering two different color images of the same object. The technique presented here can be applied to a wide variety of images. This method is computationally efficient as it only calculates the weighted linear regression line of both referenced and sensed images. This algorithm performs well for these images obtained from same sensors, having same information. But this technique falls short of for images taken from different viewpoints or from different sensors or having different information.

\section{REFERENCES}

[1] L.G.Brown. A survey of image registration techniques. ACM Comput Surv, 24(4):325-376, 1992.

[2] B. Zitova and J. Flusser. Image registration methods: a survey. Image and Vision Computing,Elsevier, 21:977-1000, 2003.

[3] R.J. Althof, M.G.J. Wind, and J.T. Dobbins. A rapid and automatic image registration algorithm with subpixel accuracy. IEEE Transactions on Medical Imaging, 16:308-316, 1997.

[4] D.I. Barnea and H.F. Silverman. A class of algorithms for fast digital image registration. IEEE Transactions on Computing, 21:179-186, 1972

[5] W.K. Pratt. Correlation techniques of image registration. IEEE Transactions on Aerospace and Electronic Systems, 10:353-358, 1974

[6] D. Skerl, B. Likar, and F. Pernus. A protocol for evaluation of similarity measures for rigid registration. IEEE Transactions On Medical Imaging, 25(6), June 2006.

[7] J. Kim and J. A. Fessler. Intensity-based image registration using robust correlation coefficients. IEEE Transactions On Medical Imaging, 23(11), November 2004.

[8] Y. Keller, A. Averbuch, and M. Israeli. Pseudo polar-based estimation of large translations, rotations, and scalings in im- ages. IEEE Trans. Image Process., vol. 14, no.1, pages 12-22, 2005.

[9] B.S. Reddy and B.N. Chatterji. An fft-based technique for translation, rotation, and scale-invariant image registration. IEEE Trans. Image Process., vol. 5, no.8, pages 1266-1271, 1996.

[10] G. Lui, J. Yan, Y. Kou, and J. Zhang. Image registration based on criteria of feature point pair mutual information. IET Image Process, 5:560-566, 2011.

[11] A. Goshtasby and G.C. Stockman. Point pattern matching using convex hull edges. IEEE Transactions on Systems, Man and Cybernetics, 15:631-637, 1985.

[12] G. Stockman, S. Kopstein, and S. Benett. Matching images to models for registration and object detection via clustering. IEEE Transactions on Pattern Analysis and Machine Intelligence, 4:229-241, 1982.

[13] W. Feng; B. Hu; C. Yang. A subpixel color image registration algorithm using quaternion phase-only correlation. IEEE, pages 1045-1049, 2008.

[14] C.D. Kuglin and D.C. Hines. The phase correlation image alignment method. IEEE Conference on Cybernetics and Society, pages 163-165, 1975 .

[15] A. Ghayoor; A. Sadri; A. Asghar; B. Shirazi. Image registration method based on physical forces for color images. Fourth Asia International Conference on Mathematical/Analytical Modelling and Computer Simulation, 2010.

[16] B.D.V. Reddy and T.J. Prasad. Color image registration and template matching using quaternion phase correlation. UbiCC, 6:714-721, 2011.

[17] J. Hazra, A. Roy Chowdhury, and P. Dutta. An approach for determining angle of rotation of a gray image using weighted statistical regression. International Journal of Scientific and Engineering Research, pages 1006 - 1013, 2013. 